\title{
Prognostic value of localization of epidermal growth factor receptor in lung adenocarcinoma
}

Jinn-Li Wang ${ }^{1,2,3}$, Chia-Lang Fang ${ }^{4,5}$, Yu-Tien Tzeng ${ }^{6}$, Han-Lin Hsu ${ }^{6,7}$, Sey-En Lin ${ }^{4,5}$, Ming-Chih Yu ${ }^{6}$, Kuan-Jen Bai ${ }^{6}$, Liang-Shun Wang ${ }^{3,8}$ and Hsingjin Eugene Liu ${ }^{3,9^{*}}$

\begin{abstract}
Background: The nuclear translocation of epidermal growth factor receptor (EGFR) has been considered to play a role in carcinogenesis. However, the relevance of differentially located EGFR proteins in lung cancer remains unclear.

Methods: We examined 161 patients with primary lung adenocarcinoma to detect EGFR expression in lung cancer cells using immunohistochemistry and determined the correlations of EGFR expression with clinical characteristics, EGFR mutations, and survival time. Moreover, we graded complete membranous staining with strong intensity as high membranous EGFR (mEGFR) expression, and nuclear EGFR staining with strong intensity as high nuclear (nEGFR) expression.
\end{abstract}

Results: The prevalence of high mEGFR and nEGFR expression in lung adenocarcinoma was 42.86 and 39.13\%, respectively. After multivariate analyses, high mEGFR expression was associated with a significantly reduced mortality risk in older patients, those with a history of smoking, and those without brain metastasis (hazard ratio[95\% confidential interval], $\mathrm{HR}[95 \% \mathrm{Cl}]=0.55[0.32 \sim 0.92] ; 0.51[0.26 \sim 0.98]$ and $0.56[0.33 \sim 0.94]$, in overall survival, respectively). An association between high nEGFR expression and early recurrence was observed in patients with metastasis ( $\mathrm{HR}[95 \% \mathrm{Cl}]=1.68[1.05 \sim 2.68]$, in progression-free survival). Notably, patients with low mEGFR and low nEGFR expression had the lowest survival rate in cases without brain metastasis $(p=0.018)$ and with a history of smoking $(p=0.062)$ and total EGFR (any high mEGFR or nEGFR) expression indicated a more favorable response to platinum-based chemotherapy regardless of EGFR mutations (HR[95\% Cl] $=0.33[0$. 12-0.92]; adjusted $\mathrm{HR}[95 \% \mathrm{Cl}]=0.36[0.13 \sim 1.02]$ with the use of tyrosine kinase inhibitor).

Conclusions: EGFR proteins at different cellular locations in lung adenocarcinoma might influence the biology of cancer cells and are an independent indicator of more favorable prognosis and treatment response.

Keywords: Epidermal growth factor receptor, Lung adenocarcinoma, Survival, Immunohistochemistry, Localization

\section{Background}

Lung cancer is the leading cause of deaths worldwide, including in Taiwan. The 5-year overall survival (OS) rate of advanced-stage lung cancer is less than 20\% [1]. Tyrosine kinase inhibitors (TKIs, erlotinib or gefitinib)

\footnotetext{
* Correspondence: liuxx086@gmail.com

${ }^{3}$ Graduate Institute of Clinical Medicine, Collage of Medicine, Taipei Medical University, 250 Wuxing St. Taipei, 11031 Taipei, Taiwan

${ }^{9}$ Division of Hematology Oncology, Department of Internal Medicine, Wan Fang Hospital, Taipei Medical University, No.111, Sec. 3, Xinglong Rd, Wenshan Dist, 11696 Taipei, Taiwan

Full list of author information is available at the end of the article
}

have been prescribed in lung adenocarcinoma patients who have epidermal growth factor receptor (EGFR) mutations and have markedly improved the survival outcome, but patients still eventually develop TKI resistance $[2,3]$. Thus, a more comprehensive understanding of lung carcinogenesis is necessary to develop more effective therapies.

Overexpression of EGFR is implicated in the pathogenesis of many human malignancies, including lung cancer [4]. EGFR overexpression has been reported to be strongly associated with cancer progression and to

(c) The Author(s). 2018 Open Access This article is distributed under the terms of the Creative Commons Attribution 4.0 International License (http://creativecommons.org/licenses/by/4.0/), which permits unrestricted use, distribution, and 
predict shorter survival in surgically resected non-small cell lung cancer (NSCLC) [5, 6]. Nevertheless, high EGFR expression may predict the response to gefitinib in lung adenocarcinoma with a high survival and provide survival benefits when gefitinib is used in combination with cetuximab in advanced NSCLC with wildtype EGFR status [7-9]. Therefore, a more detailed understanding of EGFR biology in lung carcinomas is required.

The localization of nuclear EGFR, which has been detected in various cancers in the last decade, functions as a transcription factor for cell proliferation, angiogenesis and resistance to standard therapy [10]. Nuclear EGFR expression has been reported to be related to disease progression and poor survival time in breast, ovary and oropharynx cancers as well as in early stage NSCLC [11-14]. However, few studies investigate the relationship of between EGFR proteins and EGFR mutations $[15,16]$.

Since the clinical correlation of differentially located EGFR proteins in lung cancer has not been completely evaluated; therefore, we intended to investigate the relevance of differentially located EGFR expression in lung adenocarcinoma. This study retrospectively graded differentially located EGFR expressions in 161 lung adenocarcinoma specimens by using immunohistochemistry (IHC) and determined the association with demographic characteristics, stages, EGFR mutation status, and survival time.

\section{Methods}

\section{Patients and tumor specimens}

Formalin-fixed paraffin-embedded tumors from 161 patients with lung adenocarcinoma diagnosed based on 2015 WHO classifications [17], who had undergone computed tomography-guided needle biopsies or wedge resections were collected from Taipei Medical University-Wan Fang Hospital between 2008 and 2014. The clinical parameters and follow-up data were obtained by reviewing medical records. The patients were followed until December 2016; the median follow-up period was 13.1 months (0.07-132.03). All survivors were followed for at least 12 months. This study was approved by the Joint Institutional Review Board of Taipei Medical University.

\section{Immunohistochemistry}

Four-micrometer sections of paraffin-embedded blocks were deparaffinized in xylene substitute, rehydrated with alcohol, and subjected to antigen retrieval. To detect EGFR proteins in the different cellular compartments of cancer cells, we used two types of primary EGFR antibodies to recognize EGFR proteins by the specific terminus. The mouse anti-EGFr antibody (clone 31G7, Invitrogen, Breda, the Netherlands) is raised against the NH2 terminus and recognizes membranous EGFR (mEGFR) and cytoplasmic EGFR [18]; the NCL-EGFR-384 antibody (clone EGFR.25, Novocastra, Newcastle, upon Tyne, UK) is raised against the $\mathrm{COOH}$ terminus and recognizes both mEGFR and nuclear EGFR (nEGFR) [11, 19]. Appropriate antigen retrieval protocols were used according to the specific primary antibodies. For mEGFR protein detection, we used the enzyme digestion method with pepsin (Digest-All ${ }^{\mathrm{Tm}}$ 1, Thermo Fisher Scientific) for $10 \mathrm{~min}$ at $37{ }^{\circ} \mathrm{C}$ and for nEGFR protein detection, we used the double-antigen retrieval method, heat-induced epitope retrieval (boiling citrate buffer for $5 \mathrm{~min}$ ) plus enzyme digestion with $0.025 \%$ trypsin (T4799, Sigma Aldrich) for $8 \mathrm{~min}$ at room temperature (RT). Slides were incubated at RT with the mouse anti-EGFr antibody overnight and at $4{ }^{\circ} \mathrm{C}$ with the NCL-EGFR-384 antibody overnight. The labeled streptavidin biotin method with horse radish peroxidase was used to achieve signal amplifications. Immunoreactions were visualized using 3.3'-diamino-benzidine-tetrahydrochloryte, then counterstained with hematoxylin.

\section{IHC interpretation}

mEGFR and nEGFR expression was interpreted as high and low expression from IHC images. We graded complete membranous staining with strong intensity in more than $10 \%$ of cancer cells as high mEGFR expression and others (incomplete membranous, weak or less than $10 \%$ of cancer cells staining) as low mEGFR expression. Nuclear staining with strong intensity in more than $10 \%$ of cancer cells was graded as high nEGFR expression and others were as low nEGFR expression. Lung squamous cell carcinoma tissue, known to have mEGFR overexpression, served as the positive control for mEGFR staining and hepatocellular carcinoma tissue served as the positive control for nEGFR staining. The negative controls of $\mathbf{m E G F R}$ and $\mathbf{n E G F R}$ comprised slides with the mouse IGG1 isotype antibody. The immunostain grading was done by two designated pathologists (C-L. F. and S-E. L.) with total agreement blindly. Figure 1 shows representative images for mEGFR and $\mathbf{n E G F R}$ expression.

\section{Statistical analyses}

The Pearson chi-squared test was performed to analyze the associations between differentially located EGFR proteins and clinical characteristics. The Kaplan-Meier estimate by using the log-rank test was employed to evaluate the survival distributions for differentially located EGFR proteins. OS was defined as the time between the date of diagnosis and that of death from any causes or the date of censorship (date of final 

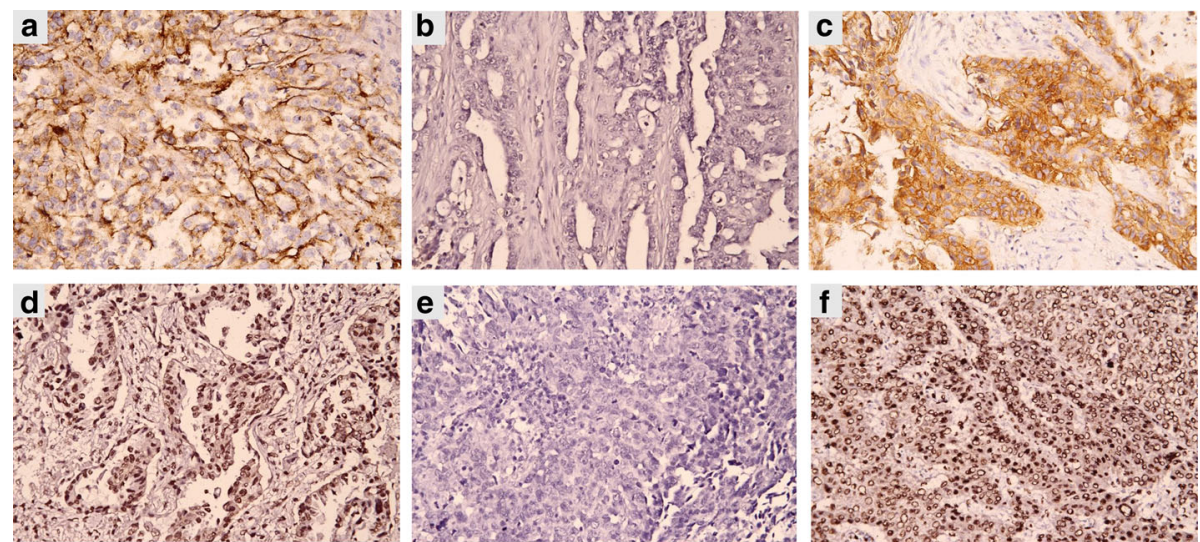

Fig. 1 Immunostaining for $\mathbf{m E G F R}$ and $\mathbf{n} E G F R$ proteins in lung adenocarcinoma tissues. a high $\mathbf{m E G F R ~ e x p r e s s i o n ; ~} \mathbf{b}$ low $\mathbf{m E G F R}$ expression; $\mathbf{c}$ positive $\mathbf{m}$ EGFR control (lung squamous cell carcinoma); $\mathbf{d}$ high $\mathbf{n E G F R ~ e x p r e s s i o n ; ~} \mathbf{e}$ low $\mathbf{n E G F R}$ expression; $\mathbf{f}$ positive $\mathbf{n}$ EGFR control (hepatocellular carcinoma). Original magnification $\times 400$

follow-up). Progression-free survival (PFS) was defined as the time between the date of treatment initiation to that of tumor progression, death from any causes, or censorship. To evaluate the mortality risk, the hazard ratio (HR) and corresponding confidence interval (CI) were estimated using Cox proportional hazards models to identify potential prognostic factors. All statistical analyses were conducted using SAS version 9.4 and as $p<0.05$ was considered statistically significant.

\section{Results}

\section{Distribution of differentially located EGFR proteins in cancer tissues}

Among the 161 lung adenocarcinoma specimens, high mEGFR and nEGFR expression was observed in 69 (42.86\%) and 63 (39.13\%) specimens, respectively. The distribution of differentially located EGFR proteins in cancer tissues had shown 36 (22.36\%) with high mEGFR and nEGFR staining, 33 (20.50\%) with high mEGFR and low nEGFR staining, 27 (16.77\%) with low mEGFR and high nEGFR staining, and 65 (40.37\%) with low mEGFR and nEGFR staining. mEGFR expression significantly correlated with nEGFR expression $(p=0.0033$, Additional file 1: Table S1). In addition, we found that morphologic characteristics were different in cancer tissues with high mEGFR expression, high nEGFR expression and EGFR mutations. The case numbers in certain subtypes of pathology were too small to draw definite conclusion. The detailed data was shown in Additional file 1: Table S2.

\section{Clinical significance of different localizations of EGFR protein}

A significantly high number of patients with low nodal stage had high mEGFR expression (odds ratio[OR, 95\% $\mathrm{CI}]=2.01[1.06-3.81], p=0.031 ;$ adjusted $\mathrm{OR}[95 \% \mathrm{CI}]=$
3.92[1.25 12.27], $\quad p=0.019$, respectively, Table 1). Compared with those with low mEGFR expression, high mEGFR expression was significantly associated with low recurrence risk in patients without brain metastasis (in univariate analyses, $\operatorname{HR}[95 \% \mathrm{CI}]=0.63[0.40-0.99]$, $p=0.045$; in multivariate analyses, $\operatorname{HR}[95 \% \mathrm{CI}]=0.56$ [0.34 0.91], $p=0.018$ in PFS) and was significantly associated with reduced mortality risk in older patients ( $>70$ years), those with a history of smoking, and those without brain metastasis (in univariate analyses, $\quad H R[95 \% \mathrm{CI}]=0.59[0.36-0.97], \quad 0.54[0.30-0.99]$ and $0.56[0.34-0.91]$ in OS, respectively; in multivariate analyses, $0.55[0.32 \sim 0.92], 0.51[0.26 \sim 0.98]$ and $0.56[0.33 \sim 0.94]$ in OS, respectively). High nEGFR expression was significantly associated with recurrence risk (in univariate analyses, $\operatorname{HR}[95 \% \mathrm{CI}]=1.58[1.01-$ 2.45] in PFS and in multivariate analyses, $\operatorname{HR}[95 \%$ $\mathrm{CI}]=1.68[1.05 \sim 2.68]$ in PFS, respectively), but did not affect mortality risk $(p=0.677)$. Notably, patients with a history of smoking who had high nEGFR expression had a significantly lower mortality risk than those who had low nEGFR expression (in univariate analyses, $\mathrm{HR}[95 \% \mathrm{CI}]=0.55[0.30-0.99]$ in OS and in multivariate analyses, $\operatorname{HR}[95 \% \mathrm{CI}]=$ $0.49[0.25 \sim 0.97]$ in OS, respectively). All data are presented in Tables 2 and 3. However, EGFR expressions (mEGFR and nEGFR) were not affected by EGFR mutations $(p=0.205$ and $p=0.734$, Table 1$)$.

\section{Survival benefits of the combination of differentially located EGFR proteins}

Since $\mathbf{n E G F R}$ proteins originate from the nuclear translocation of mEGFR proteins, we combined both immunostain types and divided them into four subgroups (mEGFR ${ }^{-}$nEGFR $^{-}, \quad \mathbf{m E G F R}{ }^{+}$nEGFR $^{-}$, $\mathbf{m E G F R}^{-} \mathbf{n E G F R}^{+}$, and $\mathbf{m E G F R}{ }^{+} \mathbf{n E G F R}^{+}$), to investigate 
Table 1 Characteristics of high membranous and nuclear EGFR expression in lung adenocarcinoma

\begin{tabular}{|c|c|c|c|c|c|c|c|}
\hline Characteristics & No & High membranous EGFR $(N=69)$ & OR $(95 \% \mathrm{Cl})$ & $P$ value & High nuclear EGFR $(N=63)$ & OR $(95 \% \mathrm{Cl})$ & $P$ value \\
\hline Age (median = $71 \mathrm{yr}$ ) & & & & 0.423 & & & 0.966 \\
\hline Younger & 77 & $30(39.0)^{\mathrm{a}}$ & 1 & & $30(38.9)$ & 1 & \\
\hline Older $>70 \mathrm{yr}$ & 84 & $39(46.4)$ & $1.36(0.73 \sim 2.54)$ & & $33(39.3)$ & $1.01(0.54 \sim 1.91)$ & \\
\hline Gender & & & & 0.436 & & & 0.692 \\
\hline Female & 90 & $41(45.6)$ & 1 & & $34(37.8)$ & 1 & \\
\hline Male & 71 & $28(39.5)$ & $0.78(0.41 \sim 1.46)$ & & $29(40.8)$ & $1.14(0.60 \sim 1.46)$ & \\
\hline Smoking & & & & 0.776 & & & 0.919 \\
\hline Never & 103 & $58(56.3)$ & 1 & & $40(38.8)$ & 1 & \\
\hline Current or past & 58 & $34(58.6)$ & $0.91(0.47 \sim 1.75)$ & & $23(39.7)$ & $1.04(0.54 \sim 1.20)$ & \\
\hline Tumor stage (2 missing) & & & & 0.584 & & & 0.455 \\
\hline $\mathrm{T} 1 / \mathrm{T} 2$ & 80 & $33(41,3)$ & 1 & & $34(42.5)$ & 1 & \\
\hline $\mathrm{T} 3 / \mathrm{T} 4$ & 79 & $36(45.6)$ & $1.19(0.64 \sim 2.23)$ & & $29(36.7)$ & $0.78(0.42 \sim 1.48)$ & \\
\hline Nodal stage (1 missing) & & & & 0.031 & & & 0.466 \\
\hline LO/L1 & 68 & $36(52.9)$ & $2.01(1.06 \sim 3.81)^{b}$ & & $29(42.6)$ & $1.27(0.67 \sim 2.41)$ & \\
\hline L2/L3 & 92 & $33(35.9)$ & 1 & & $34(36.9)$ & 1 & \\
\hline Metastasis & & & & 0.090 & & & 0.118 \\
\hline without & 67 & $34(50.7)$ & 1 & & $31(46.3)$ & 1 & \\
\hline with & 94 & $35(37.2)$ & $0.58(0.30 \sim 1.09)$ & & $32(34.0)$ & $0.60(0.32 \sim 1.14)$ & \\
\hline TNM stage & & & & 0.045 & & & 0.247 \\
\hline Localized (stage I/II) & 43 & $24(55.8)$ & $2.05(1.01 \sim 4.16)^{c}$ & & $20(46.5)$ & $1.52(0.75 \sim 3.08)$ & \\
\hline Distant (stage III/IV) & 118 & $45(38.1)$ & 1 & & $43(36.4)$ & 1 & \\
\hline Brain metastasis & & & & 0.515 & & & 0.890 \\
\hline without & 114 & $47(41.2)$ & 1 & & $45(39.5)$ & 1 & \\
\hline with & 47 & $22(46.8)$ & $1.25(0.63 \sim 2.49)$ & & $18(38.3)$ & $0.95(0.47 \sim 1.91)$ & \\
\hline EGFR mutations $^{d}$ & & & & 0.205 & & & 0.634 \\
\hline Wildtype & 74 & $28(37.8)$ & 1 & & $27(36.5)$ & 1 & \\
\hline Mutations ${ }^{\mathrm{e}}$ & 77 & $37(48.0)$ & $1.52(0.79 \sim 2.91)$ & & $31(40.3)$ & $1.17(0.61 \sim 2.26)$ & \\
\hline
\end{tabular}

Chi-squared test

${ }^{\mathrm{a}}$ Data are presented as $\mathrm{n}(\%)$ of row

${ }^{\mathrm{b}}$ Adjusted OR $(95 \% \mathrm{Cl})=3.92(1.25 \sim 12.27), p=0.019$

${ }^{c}$ Adjusted OR $(95 \% \mathrm{Cl})=1.37(0.32 \sim 5.98) p=0.674$

${ }^{d} 10$ missing

e Including 3 in exon 18, 37 in exon 19, 3 in exon 20, 33 in exon 21and 1 in exon 19/20

Note: Boldfaces as statistical significance

Table 2 Univariate analyses for progression-free and overall survival in lung adenocarcinoma

\begin{tabular}{|c|c|c|c|c|c|c|c|c|c|}
\hline \multirow{3}{*}{$\begin{array}{l}\text { Variable } \\
\text { Parameter }\end{array}$} & \multirow{3}{*}{ No } & \multicolumn{4}{|c|}{ High membranous EGFR } & \multicolumn{4}{|c|}{ High nuclear EGFR } \\
\hline & & \multicolumn{2}{|l|}{$\overline{\mathrm{PFS}}$} & \multicolumn{2}{|l|}{ OS } & \multicolumn{2}{|l|}{ PFS } & \multicolumn{2}{|l|}{ OS } \\
\hline & & ${ }^{a} \mathrm{HR}(95 \% \mathrm{Cl})$ & $p$ & ${ }^{a} \mathrm{HR}(95 \% \mathrm{Cl})$ & $p$ & bHR $(95 \% \mathrm{Cl})$ & $p$ & ${ }^{\mathrm{b}} \mathrm{HR}(95 \% \mathrm{Cl})$ & $p$ \\
\hline All patients & 161 & $0.79(0.56 \sim 1.13)$ & 0.198 & $0.71(0.49 \sim 1.03)$ & 0.067 & $1.03(0.73 \sim 1.46)$ & 0.865 & $0.81(0.56 \sim 1.17)$ & 0.251 \\
\hline Older age (> 70 yr) & 84 & $0.64(0.39 \sim 1.04)$ & 0.068 & $0.59(0.36 \sim 0.97)$ & 0.038 & $0.81(0.50 \sim 1.31)$ & 0.394 & $0.69(0.40 \sim 1.10)$ & 0.101 \\
\hline Smokers & 58 & $0.62(0.35 \sim 1.09)$ & 0.092 & $0.54(0.30 \sim 0.99)$ & 0.046 & $0.61(0.34 \sim 1.10)$ & 0.102 & $0.55(0.30 \sim 0.99)$ & 0.043 \\
\hline Distant (metastasis) & 94 & $1.01(0.66 \sim 1.56)$ & 0.954 & $0.83(0.54 \sim 1.29)$ & 0.403 & $1.58(1.01 \sim 2.45)$ & 0.043 & $1.10(0.70 \sim 1.73)$ & 0.677 \\
\hline No brain metastasis & 114 & $0.63(0.40 \sim 0.99)$ & 0.045 & $0.56(0.34 \sim 0.91)$ & 0.019 & $1.01(0.66 \sim 1.56)$ & 0.953 & $0.78(0.49 \sim 1.25)$ & 0.297 \\
\hline Wildtype EGFR & 74 & $0.60(0.35 \sim 1.03)$ & 0.059 & $0.64(0.37 \sim 1.09)$ & 0.094 & $0.84(0.50 \sim 1.41)$ & 0.506 & $0.74(0.43 \sim 1.26)$ & 0.265 \\
\hline
\end{tabular}

Cox proportional hazards model

a Low membranous EGFR expression as a reference

bLow nuclear EGFR expression as a reference

Note: Boldfaces as statistical significance 
Table 3 Multivariate analyses for progression-free and overall survival in lung adenocarcinoma

\begin{tabular}{|c|c|c|c|c|c|c|c|c|c|}
\hline \multirow{3}{*}{$\begin{array}{l}\text { Variable } \\
\text { Parameter }\end{array}$} & \multirow{3}{*}{ No } & \multicolumn{4}{|c|}{ High membranous EGFR } & \multicolumn{4}{|l|}{ High nuclear EGFR } \\
\hline & & \multicolumn{2}{|l|}{ PFS } & \multicolumn{2}{|l|}{ OS } & \multicolumn{2}{|l|}{ PFS } & \multicolumn{2}{|l|}{ OS } \\
\hline & & ${ }^{\mathrm{a}} \mathrm{HR}(95 \% \mathrm{Cl})$ & $p$ & ${ }^{\mathrm{a}} \mathrm{HR}(95 \% \mathrm{Cl})$ & $p$ & ${ }^{\mathrm{b}} \mathrm{HR}(95 \% \mathrm{Cl})$ & $p$ & ${ }^{\mathrm{b}} \mathrm{HR}(95 \% \mathrm{Cl})$ & $p$ \\
\hline All patients & 161 & $0.72(0.50 \sim 1.04)$ & 0.078 & $0.70(0.47 \sim 1.03)$ & 0.071 & $0.89(0.61 \sim 1.29)$ & 0.529 & $0.72(0.49 \sim 1.07)$ & 0.103 \\
\hline Older age (> 70 yr) & 84 & $0.60(0.36 \sim 0.99)$ & 0.046 & $0.55(0.32 \sim 0.92)$ & 0.023 & $0.77(0.47 \sim 1.27)$ & 0.310 & $0.62(0.37 \sim 1.05)$ & 0.077 \\
\hline Smokers & 58 & $0.63(0.34 \sim 1.16)$ & 0.135 & $0.51(0.26 \sim 0.98)$ & 0.045 & $0.64(0.34 \sim 1.21)$ & 0.166 & $0.49(0.25 \sim 0.97)$ & 0.041 \\
\hline istant (metastasis) & 94 & $1.20(0.76 \sim 1.88)$ & 0.434 & $1.00(0.63 \sim 1.61)$ & 0.985 & $1.68(1.05 \sim 2.68)$ & 0.030 & $1.27(0.77 \sim 2.08)$ & 0.350 \\
\hline No brain metastasis & 114 & $0.56(0.34 \sim 0.91)$ & 0.018 & $0.56(0.33 \sim 0.94)$ & 0.028 & $0.86(0.54 \sim 1.36)$ & 0.521 & $0.72(0.44 \sim 1.18)$ & 0.186 \\
\hline Wildtype EGFR & 74 & $0.49(0.28 \sim 0.85)$ & 0.012 & $0.51(0.29 \sim 0.90)$ & 0.019 & $0.73(0.43 \sim 1.24)$ & 0.240 & $0.62(0.36 \sim 1.08)$ & 0.092 \\
\hline
\end{tabular}

Cox proportional hazards model after adjustment of other variables except distant

aLow membranous EGFR expression as a reference

bow nuclear EGFR expression as a reference

Note: Boldfaces as statistical significance

the synergistic effects on survival outcome. Although we did not find any survival differences among all patients ( $p=0.112$, data not shown), patients with low mEGFR and nEGFR expression had the lowest survival rate among patients without brain metastasis and with a history of smoking $(p=0.018$ and 0.062 , respectively, Fig. 2a and b).

Next, we considered high mEGFR and nEGFR expression together as total EGFR (tEGFR) expression, defined as either high $\mathbf{m E G F R}$ or $\mathbf{n E G F R}$ expression, to investigate the treatment response in patients receiving different anti-cancer therapies. tEGFR expression was significantly associated with a reduced mortality risk in patients with a history of smoking and without brain metastasis (HR[95\% $\mathrm{CI}]=0.24[0.07-0.81], p=0.013 ; \mathrm{HR}[95 \% \mathrm{CI}]=0.43[0.18-$ 1.06], $p=0.045$, respectively) who were receiving platinum-based chemotherapy, as well as in patients without brain metastasis (HR $[95 \% \mathrm{CI}]=0.45[0.20-0.98], p=$ 0.040) after EGFR TKI use. All data are shown in Table 4.

\section{Synergistic effect of tEGFR protein and EGFR mutations on overall survival}

Based on lung adenocarcinoma patients with EGFR mutations responsible to EGFR TKIs, we compared the combined effects of tEGFR protein and EGFR mutations on clinical benefits. Univariate analysis had shown the survival difference ( $p=0.001$, Fig. 2c) in four subgroups $\left(\right.$ tEGFR $^{-}$mutant $^{-}$, tEGFR $^{+}$mutant $^{-}$, tEGFR $^{-}$mutant $^{+}$ and $\mathrm{tEGFR}^{+}$mutant $^{+}$). Then we examined the treatment response to platinum-based chemotherapy and found that comparing with patients without any biomarker $\left(\right.$ tEGFR $^{-}$mutant $\left.^{-}\right)$, tEGFR protein was significantly associated with low mortality risk $(\mathrm{HR}[95 \% \mathrm{CI}]=0.33[0.12$ 0.92], $p=0.029$; adjusted $\mathrm{HR}[95 \% \mathrm{CI}]=0.36[0.13 \sim 1.02]$, $p=0.055$, with the use of TKI). All data are shown in Table 5.

\section{Discussion}

This study investigated differentially located EGFR expression in lung adenocarcinoma. Our data indicate that high mEGFR expression is a more favorable prognostic factor in older patients, those with a history of smoking, and those without brain metastasis. Moreover, high nEGFR expression predicts early relapse in patients with distant metastasis. Notably, the combination of mEGFR and $\mathbf{n E G F R}$ expression is associated with survival benefits and with a more favorable response to anti-cancer therapies in patients with a history of smoking and
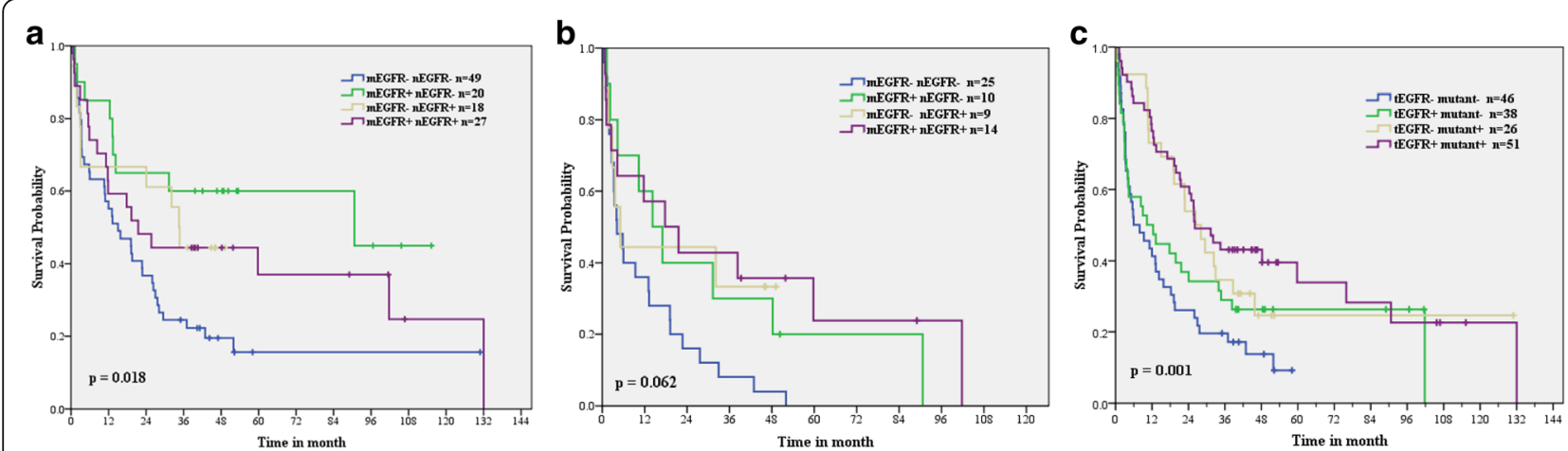

Fig. 2 Survival analyses. a patients without brain metastasis and $\mathbf{b}$ patients with a history of smoking, stratified by high $\mathbf{m E G F R}$ and $\mathbf{n} E G F R$ expression; $\mathbf{c}$ total patients stratified by $\mathbf{t E G F R}$ expression and EGFR mutations 
Table 4 Comparisons of treatment responses according to tEGFR expression

\begin{tabular}{ccllll}
\hline Parameters & No & tEGFR & Median $^{\mathrm{a}}(\mathrm{m})$ & HR $(95 \% \mathrm{Cl})$ & $P$ value \\
\hline Smokers & & & & & \\
Platinum & 9 & Positive & 59.8 & $0.24(0.07 \sim 0.81)$ & $\mathbf{0 . 0 1 3}$ \\
& 8 & Negative & 20.9 & 1 & \\
TKls & 9 & Positive & 32.1 & $0.40(0.12 \sim 1.33)$ & 0.122 \\
& 6 & Negative & 16.2 & 1 & \\
Radiation & 9 & Positive & 17.0 & $0.56(0.21 \sim 1.53)$ & 0.253 \\
& 11 & Negative & 13.1 & 1 &
\end{tabular}

No brain metastasis

\begin{tabular}{llllll} 
Platinum & 18 & Positive & 59.8 & $0.43(0.18 \sim ~ 1.06)$ & $\mathbf{0 . 0 4 5}$ \\
& 14 & Negative & 23.4 & 1 & \\
TKls & 20 & Positive & 33.3 & $0.45(0.20 \sim 0.98)$ & $\mathbf{0 . 0 4 0}$ \\
& 16 & Negative & 17.5 & 1 & \\
Radiation & 14 & Positive & 58.2 & $0.55(0.20 \sim 1.48)$ & 0.230 \\
& 11 & Negative & 12.3 & 1 & \\
\hline
\end{tabular}

${ }^{a}$ Median $=$ median survival time

Note: Boldfaces as statistical significance

without brain metastasis. Therefore, we suggest that differentially located EGFR expression synergistically predict survival outcomes and treatment responses in lung adenocarcinoma patients.

In this study, a high number of patients with low nodal stage exhibited high mEGFR expression, possibly indicating the initial stage of lung carcinogenesis. These results are different from those obtained in previous studies, which have reported a higher prevalence of EGFR overexpression in tumors of advanced stage and with lymph node invasion in colon and pancreatic cancer as well as in early stage (IA to IIIA) NSCLC $[5,20,21]$. Nevertheless, we did not observe any clinical associations for $\mathbf{n E G F R}$ proteins, although nEGFR has been associated with higher-stage breast cancer and higher disease stage in early-stage NSCLC [14, 19]. Such differences might have been a result of most enrolled patients having advanced-stage lung adenocarcinoma.

In accordance with recent studies on breast, ovarian and head-and-neck cancers, which have reported the prognostic value of $\mathbf{n E G F R}$ proteins for survival outcomes [12, 13, 19], the role of $\mathbf{n E G F R}$ expression in predicting recurrence risk in the metastasis subgroup was addressed in this study. However, clinicians may provide multi-agent therapies to patients with lung cancer relapse; therefore, the survival outcomes in patients with metastasis exhibited no differences. Altogether, we suggest that adjusting clinical management according to $\mathbf{n E G F R}$ expression at initial diagnosis might reduce early recurrence risk in patients with advanced lung adenocarcinoma.

In contrast to previous studies that EGFR overexpression has been associated with poor survival prognosis $[5,6,22]$, this study has determined the survival benefits of differentially located EGFR proteins in those who had a history of smoking and no brain metastasis by observing more favorable treatment responses in patients with tEGFR expression. Although we could not exclude the effects of EGFR mutations on anti-EGFR therapies, we had found that patients with tEGFR protein was responsible to platinum-based chemotherapy regardless of EGFR mutations by observing the $\mathrm{tEGFR}^{+}$mutant $^{-}$subgroup with significantly less mortality risk than the tEGFR $^{-}$mutant ${ }^{-}$subgroup. Our findings are in accordance with a recent Chinese study that IHC positive mEGFR expression is associated with responses to EGFR TKIs in NSCLC patients with wildtype EGFR status [23]. Targeting EGFR protein has been reported to be an important treatment option for NSCLC [24]; therefore, EGFR proteins might be an indicator for treatment responses in patients with lung adenocarcinoma. However, the true mechanism warrants further investigation.

\section{Conclusions}

This present study indicated that differentially located EGFR proteins might serve as a molecular marker of survival outcomes in patients with lung adenocarcinoma. Since EGFR proteins were responsible to platinum-based chemotherapy, treatment selection according to EGFR expression might be essential in the treatment of lung adenocarcinoma patients. Prospective studies are required to validate our theory.

Table 5 Hazard ratios for overall survival in the joint subgroups with platinum-based chemotherapy

\begin{tabular}{llllll}
\hline tEGFR/mutant & Number & Median $(\mathrm{m})^{\mathrm{a}}$ & Unadjusted HR $(95 \% \mathrm{Cl})$ & $p$ & Adjusted HR $^{\mathrm{b}}(95 \% \mathrm{Cl})$ \\
\hline tEGFR $^{-}$mutant $^{-}$ & 11 & 18.2 & 1.0 & & 1.0 \\
tEGFR $^{+}$mutant $^{-}$ & 10 & 34.7 & $0.33(0.12 \sim 0.92)$ & $\mathbf{0 . 0 2 9}$ & $0.36(0.13 \sim 1.02)$ \\
tEGFR $^{-}$mutant $^{+}$ & 10 & 29.5 & $0.65(0.41 \sim 1.04)$ & 0.070 & $0.64(0.39 \sim 1.05)$ \\
tEGFR $^{+}$mutant $^{+}$ & 21 & 25.9 & $0.74(0.56 \sim 0.97)$ & $\mathbf{0 . 0 3 3}$ & $0.83(0.59 \sim 1.15)$ \\
\hline
\end{tabular}

Cox proportional model

${ }^{\mathrm{a}}$ Median $(\mathrm{m})=$ median survival time (month)

${ }^{\mathrm{b}}$ Adjusted hazard ratio with TKI

Note: Boldfaces as statistical significance 


\section{Additional file}

Additional file 1 : Table S1. Distribution of differentially located EGFR expression in lung adenocarcinoma and Table S2. Morphologic characteristics for $\mathbf{m E G F R}$ expression, $\mathbf{n E G F R}$ expression and EGFR mutations in lung adenocarcinoma. (DOCX $16 \mathrm{~kb}$ )

\section{Abbreviations}

Cl: Confidence interval; EGFR: Epidermal growth factor receptor; HR: Hazard ratio; IHC: Immunohistochemistry; NSCLC: Non-small cell lung cancer; OR: Odds ratio; OS: Overall survival; PFS: Progression-free survival; TKl: Tyrosine kinase inhibitor

\section{Acknowledgements}

The authors thank the study participants, medical staff for patient' care, and Prof. Mey Wang for statistical analyses. This study was supported in parts by the grants from Wan Fang Hospital (103-wf-phd-03 and 104-wf-phd-03), Taiwan. This manuscript was edited by Wallace Academic Editing.

\section{Funding}

103-wf-phd-03 and 104-wf-phd-03 from WanFang Hospital.

\section{Availability of data and materials}

All data generated or analyzed during this study are included in this published article (and its Additional files).

\section{Authors' contributions}

JLW designed this study, analyzed the patient data, and wrote the manuscript; CLF and SEL interpreted and graded the IHC images; YTT, HLH, MCY, KJB and LSW collected the patient data; HEJ revised the manuscript. All authors read and approved the final manuscript.

\section{Ethics approval and consent to participate}

This study had been approved by the Joint Institutional Review Board of Taipei Medical University.

\section{Competing interests}

The authors declare that they have no competing interests.

\section{Publisher's Note}

Springer Nature remains neutral with regard to jurisdictional claims in published maps and institutional affiliations.

\section{Author details}

${ }^{1}$ Division of Hematology Oncology, Department of Pediatrics, Wan Fang Hospital, Taipei Medical University, No.111, Sec. 3, Xinglong Rd, Wenshan Dist, 11696 Taipei, Taiwan. ${ }^{2}$ Department of Pediatrics, School of Medicine, College of Medicine, Taipei Medical University, 250 Wuxing St. Taipei, 11031 Taipei, Taiwan. ${ }^{3}$ Graduate Institute of Clinical Medicine, Collage of Medicine, Taipei Medical University, 250 Wuxing St. Taipei, 11031 Taipei, Taiwan. ${ }^{4}$ Department of Pathology, Wan Fang Hospital, Taipei Medical University, No.111, Sec. 3, Xinglong Rd, Wenshan Dist, 11696 Taipei, Taiwan. ${ }^{5}$ Department of Pathology, School of Medicine, College of Medicine, Taipei Medical University, 250 Wuxing St. Taipei, 11031 Taipei, Taiwan. ${ }^{6}$ Division of Pulmonary Medicine, Department of Internal Medicine, Wan Fang Hospital, Taipei Medical University, No.111, Sec. 3, Xinglong Rd, Wenshan Dist, 11696 Taipei, Taiwan. ${ }^{7}$ Graduate Institute of Medical Sciences, College of Medicine, Taipei Medical University, 250 Wuxing St. Taipei, 11031 Taipei, Taiwan. ${ }^{8}$ Division of Thoracic Surgery, Department of Surgery, Shuang Ho Hospital, Taipei Medical University, No.291, Zhongzheng Rd., Zhonghe District, New Taipei City 23561, Taiwan. ${ }^{9}$ Division of Hematology Oncology, Department of Internal Medicine, Wan Fang Hospital, Taipei Medical University, No.111, Sec. 3, Xinglong Rd, Wenshan Dist, 11696 Taipei, Taiwan.
Received: 13 February 2018 Accepted: 22 May 2018

Published online: 28 June 2018

\section{References}

1. Siegel RL, Miller KD, Jemal A. Cancer statistics, 2016. CA Cancer J Clin. 2016; 66:7-30.

2. Paez JG, Janne PA, Lee JC, Tracy S, Greulich H, Gabriel S, et al. EGFR mutations in lung cancer: correlation with clinical response to gefitinib therapy. Science. 2004;304:1497-500.

3. Maemondo M, Inoue A, Kobayashi K, Sugawara S, Oizumi S, Isobe H, et al. Gefitinib or chemotherapy for non-small-cell lung cancer with mutated EGFR. N Engl J Med. 2010;362:2380-8

4. Bethune $\mathrm{G}$, Bethune D, Ridgway N, Xu Z. Epidermal growth factor receptor (EGFR) in lung cancer: an overview and update. J Thoracic Dis. 2010;2:48-51.

5. Selvaggi G, Novello S, Torri V, Leonardo E, De Giuli P, Borasio P, et al. Epidermal growth factor receptor overexpression correlates with a poor prognosis in completely resected non-small-cell lung cancer. Ann Oncol. 2004;15:28-32.

6. Ohsaki Y, Tanno S, Fujita Y, Toyoshima E, Fujiuchi S, Nishigaki Y, et al. Epidermal growth factor receptor expression correlates with poor prognosis in non-small cell lung cancer patients with p53 overexpression. Oncol Rep. 2000;7:603-7.

7. Gupta R, Dastane AM, McKenna R Jr, Marchevsky AM. The predictive value of epidermal growth factor receptor tests in patients with pulmonary adenocarcinoma: review of current "best evidence" with meta-analysis. Hum Pathol. 2009:40:356-65.

8. Douillard JY, Pirker R, O'Byrne KJ, Kerr KM, Storkel S, von Heydebreck A, et al. Relationship between EGFR expression, EGFR mutation status, and the efficacy of chemotherapy plus cetuximab in FLEX study patients with advanced non-small-cell lung cancer. J Thorac Oncol. 2014;9:717-24.

9. Cappuzzo F, Hirsch FR, Rossi E, Bartolini S, Ceresoli GL, Bemis L, et al. Epidermal growth factor receptor gene and protein and gefitinib sensitivity in non-small-cell lung cancer. J Natl Cancer Inst. 2005;97:643-55.

10. Brand TM, lida M, Li C, Wheeler DL. The nuclear epidermal growth factor receptor signaling network and its role in cancer. Discov Med. 2011;12:41932

11. Lo HW, Xia W, Wei Y, Ali-Seyed M, Huang SF, Hung MC. Novel prognostic value of nuclear epidermal growth factor receptor in breast cancer. Cancer Res. 2005;65:338-48.

12. Xia W, Wei Y, Du Y, Liu J, Chang B, Yu YL, et al. Nuclear expression of epidermal growth factor receptor is a novel prognostic value in patients with ovarian cancer. Mol Carcinog. 2009;48:610-7.

13. Psyrri A, Yu Z, Weinberger PM, Sasaki C, Haffty B, Camp R, et al. Quantitative determination of nuclear and cytoplasmic epidermal growth factor receptor expression in oropharyngeal squamous cell cancer by using automated quantitative analysis. Clin Cancer Res. 2005;11:5856-62.

14. Traynor AM, Weigel TL, Oettel KR, Yang DT, Zhang C, Kim K, et al. Nuclear EGFR protein expression predicts poor survival in early stage non-small cell lung cancer. Lung Cancer. 2013;81:138-41.

15. Helfrich BA, Raben D, Varella-Garcia M, Gustafson D, Chan DC, Bemis L, et al. Antitumor activity of the epidermal growth factor receptor (EGFR) tyrosine kinase inhibitor gefitinib (ZD1839, Iressa) in non-small cell lung cancer cell lines correlates with gene copy number and EGFR mutations but not EGFR protein levels. Clin Cancer Res. 2006;12:7117-25.

16. Xu JM, Han Y, Duan HQ Gao EM, Zhang Y, Liu XQ et al. EGFR mutations and HER2/3 protein expression and clinical outcome in Chinese advanced non-small cell lung cancer patients treated with gefitinib. J Cancer Res Clin Oncol. 2009;135:771-82.

17. Inamura K. Lung Cancer: understanding its molecular pathology and the 2015 WHO classification. Front Oncol. 2017;7:193.

18. Schrevel M, Gorter A, Kolkman-Uljee SM, Trimbos JB, Fleuren GJ, Jordanova ES. Molecular mechanisms of epidermal growth factor receptor overexpression in patients with cervical cancer. Mod Pathol. 2011;24:720-8.

19. Hadzisejdic I, Mustac E, Jonjic N, Petkovic M, Grahovac B. Nuclear EGFR in ductal invasive breast cancer: correlation with cyclin-D1 and prognosis. Mod Pathol. 2010:23:392-403.

20. Lockhart AC, Berlin JD. The epidermal growth factor receptor as a target for colorectal cancer therapy. Semin Oncol. 2005:32:52-60.

21. Mahipal A, McDonald MJ, Witkiewicz A, Carr BI. Cell membrane and cytoplasmic epidermal growth factor receptor expression in pancreatic ductal adenocarcinoma. Med Oncol. 2012;29:134-9. 
22. Psyrri A, Kassar M, Yu Z, Bamias A, Weinberger PM, Markakis S, et al. Effect of epidermal growth factor receptor expression level on survival in patients with epithelial ovarian cancer. Clin Cancer Res. 2005;11:8637-43.

23. Xu N, Fang W, Mu L, Tang Y, Gao L, Ren S, et al. Overexpression of wildtype EGFR is tumorigenic and denotes a therapeutic target in non-small cell lung cancer. Oncotarget. 2016;7:3884-96.

24. Al Olayan A, Al Hussaini $H$, Jazieh AR. The roles of epidermal growth factor receptor (EGFR) inhibitors in the management of lung cancer. J Infect Public Health. 2012;5(Suppl 1):S50-60.

Ready to submit your research? Choose BMC and benefit from:

- fast, convenient online submission

- thorough peer review by experienced researchers in your field

- rapid publication on acceptance

- support for research data, including large and complex data types

- gold Open Access which fosters wider collaboration and increased citations

- maximum visibility for your research: over $100 \mathrm{M}$ website views per year 